\title{
Transfert d'oxygène et anoxie dans les sols agrégés : contribution à l'étude de la dénitrification
}

\author{
P Renault ${ }^{1 *}$, J Sierra 2 , P Stengel 1 \\ 1 INRA, unité de science du sol, domaine Saint-Paul, BP 91, F84143 Montfavet cedex, France ; \\ 2 Departemento de Suelos, Facultad de Agronomia, Universidad de Buenos Aires, 1417 Buenos Aires, Argentine
}

(Reçu le 12 avril 1994; accepté le $1^{\text {er }}$ août 1994)

\begin{abstract}
Résumé - L'anoxie dans les sols à structure agrégée a été étudiée afin de mieux cerner les déterminants physiques de la dénitrification. Un modèle numérique simulant les transferts d'oxygène nous a permis d'estimer l'anoxie dans des agrégats homogènes de forme et de dimension variées, présentant éventuellement des occlusions au niveau de leur surface externe. Ces occlusions correspondent aux contacts entre les agrégats et à leur recouvrement partiel par de l'eau. L'anoxie au sein des agrégats peut être approchée à l'aide d'un modèle simplifié. Couplé à un modèle de transfert d'oxygène dans l'espace poral interagrégats, ce modèle aboutit, à l'échelle du profil cultural, à des relations entre l'anoxie et la teneur en eau qui sont similaires aux relations proposées dans la littérature entre la dénitrification et la teneur en eau. Ce modèle met en évidence des interactions entre les effets de la teneur en eau, de la température et de la structure. Des résultats expérimentaux ont été obtenus au moyen de la chromatographie en phase gazeuse et de micro-électrodes à oxygène. La respiration microbienne dépend des concentrations en oxygène et en dioxyde de carbone. Ce dernier l'inhibe généralement mais peut l'activer à faible concentration. II existe une grande variabilité de la respiration globale des agrégats naturels. La distribution de l'oxygène est irrégulière en leur sein, en raison de la distribution hétérogène de l'espace poral et probablement de la matière organique. En ajoutant au modèle le transport du dioxyde de carbone et l'effet de l'oxygène sur la respiration, il est possible de retrouver approximativement la respiration globale des agrégats de sol remanié et la distribution de l'oxygène en leur sein.
\end{abstract}

anaérobiose dans les sols / diffusion gazeuse / respiration microbienne / modélisation numérique / microélectrode à oxygène

Summary - Oxygen transport and anaerobiosis in aggregated soils: contribution to the study of denitrification. Anaerobiosis was studied in aggregated soils in order to improve the knowledge of physical factors affecting denitrification. A numerical model was used to simulate oxygen transport and anaerobiosis in homogeneous aggregates of various shapes and dimensions, with possible occlusions of some parts of their external surface. These occlusions are due to contacts with neighbouring aggregates and to water menisci around them. It is possible to obtain similar results using an empirical model. By using this model and a model describing oxygen diffusion in the inter-crumb pore space we obtained relationships between anaerobiosis and water content similar to previously proposed empirical relationships between denitrification and water content. The model showed interactions between the effects of water content, temperature and soil structure. Experiments were carried out using gas chromatography and oxygen microelectrodes. Microbial respiration depends on oxygen and carbon dioxide concentrations. Carbon dioxide generally inhibits respiration but may increase it at low level. There is a large variability in global respiration of natural aggregates and oxygen concentration distribution is irregular inside them, as a consequence of the distribution of the pore space and probably the distribution of the organic matter. For aggregates of remoulded soil, it is possible to simulate their global respiration and the oxygen concentration distribution inside, when accounting for carbon dioxide transport and oxygen effect on microbial respiration.

\section{soil anaerobiosis / gaseous diffusion / microbial respiration / numerical modelling / oxygen microelectrode}

\footnotetext{
* Correspondance et tirés à part
} 


\section{INTRODUCTION}

Il est bien connu que l'activité microbienne d'un sol est fortement dépendante de sa teneur en eau. Aux faibles teneurs en eau, l'activité microbienne peut être réduite en raison du potentiel de l'eau, de la teneur en eau elle-même ou de la diffusion de plusieurs molécules et ions (Skopp et al, 1990). Aux fortes teneurs en eau, alors que ces facteurs peuvent être sans importance, la diffusion des gaz peut devenir insuffisante pour fournir suffisamment d' $\mathrm{O}_{2}$ aux bactéries (Leffelaar, 1979 ; Smith, 1980 ; Arah et Smith, 1989). Dans ces conditions, des bactéries anaérobies facultatives peuvent substituer les ions $\mathrm{NO}_{3}{ }^{-}$ou $\mathrm{NO}_{2}{ }^{-}$à $\mathrm{l}^{\prime} \mathrm{O}_{2}$ comme accepteur final d'électrons dans les réactions respiratoires (Knowles, 1982 ; Firestone, 1982). Dans ce processus, $\mathrm{NO}_{3}^{-}$ou $\mathrm{NO}_{2}{ }^{-}$sont généralement réduits en $\mathrm{N}_{2} \mathrm{O}$ ou en $\mathrm{N}_{2}$ rejetés dans l'atmosphère (processus de dénitrification). Parfois, le $\mathrm{NO}_{3}{ }^{-}$ou le $\mathrm{NO}_{2}{ }^{-}$peuvent être aussi réduits en $\mathrm{NH}_{4}{ }^{+}$de manière dissimilative (Knowles, 1982 ; Fazzolari et al, 1990a et 1990b).

Plusieurs chercheurs ont utilisé des relations empiriques dépendant de la teneur en eau pour modéliser les transformations biologiques de l'azote et du carbone dans le sol (Bridge et Rixon, 1976 ; Rolston et al, 1984 ; Linn et Doran, 1984 ; Clay et al, 1985 ; Grundmann et Rolston, 1987 ; Johnsson et al, 1987 ; Vereecken et al, 1991), mais les possibilités d'application de ces fonctions peuvent être limitées aux conditions de sol dans lesquelles elles furent obtenues (Grant, 1991). Skopp et al (1990) ont modélisé l'activité microbienne du sol par une fonction dépendant de la teneur en eau et de la porosité en considérant plusieurs processus dans le sol (diffusion de $\mathrm{I}^{\prime} \mathrm{O}_{2}$ et des substrats). Ces fonctions de la teneur en eau sont reliées au taux de saturation en eau du sol, à sa teneur en eau volumique ou au potentiel hydrique. Elles décrivent l'activité microbienne en relation à une activité de référence qui dépend elle-même d'autres paramètres (concentration en $\mathrm{NO}_{3}^{-}$, teneur en matières organiques, température du sol). Plusieurs de ces fonctions ont été utilisées pour décrire la dénitrification. Ces dernières sont maximales à la saturation et prennent une valeur nulle en dessous d'une teneur en eau critique comprise généralement entre $60 \%$ et $80 \%$ de la teneur en eau à saturation (Aulakh et al, 1982 ; Rolston et al, 1984 ; Grundmann et Rolston, 1987 ; Sexstone et al, 1988). Vereecken et al (1991) ont supposé que l'activité dénitrifiante existait à toutes les teneurs en eau, y compris les plus faibles.

L'influence de la température, des concentrations en $\mathrm{NO}_{3}{ }^{-}$et en $\mathrm{NH}_{4}{ }^{+}$, de la teneur en matières organiques ont généralement été prises en compte en introduisant des fonctions multiplicatives (Rolston et al, 1984 ; Clay et al, 1985 ; Grundmann et Rolston, 1987). Ce faisant, les chercheurs ont implicitement supposé qu'il n'y a pas d'interactions entre les paramètres ou que ces interactions pouvaient être négligées. En fait, des interactions peuvent exister (Groot et De Willingen, 1991). Par exemple, la température agit simultanément de différentes façons. Aux fortes teneurs en eau, elle influence la solubilité de l' $\mathrm{O}_{2}$ dans l'eau, la diffusion de l' $\mathrm{O}_{2}$ dans l'air et dans l'eau et l'activité microbienne (Skopp, 1985 ; Renault et Stengel, 1994). Pour la dénitrification, un changement de température ne modifie pas seulement l'activité microbienne dans les régions anoxiques, mais aussi la fraction volumique de ces régions dans le sol. Grant (1991) considérait les effets simultanés de la température sur l'activité microbienne et la solubilité de $\mathrm{l}^{\prime} \mathrm{O}_{2}$. Les réponses qu'il a simulées indiquent une interaction entre la teneur en eau du sol et la température : lorsque la température décroît, la teneur en eau minimale pour qu'it y ait dénitrification croît.

Sans aucun doute, l'estimation expérimentale des fonctions microbiologiques est difficile en raison de la variété des facteurs affectant l'activité microbienne. C'est probablement la raison pour laquelle plusieurs auteurs ont utilisé la même fonction, quel que soit le type de sol et sa structure (Hansen et al, 1991). Cependant, la structure du sol affecte beaucoup le transport de $\mathrm{I}^{\prime} \mathrm{O}_{2}$ et sa fraction en état d'anoxie (Greenwood et Berry, 1962 ; Smith, 1980 ; Renault et Stengel, 1994). Le dernier problème concerne les échelles variées d'intérêt d'espace et de temps (McConnaughey et Bouldin, 1985). En particulier, plusieurs fonctions relatives à la dénitrification ont été établies à l'échelle de la parcelle cultivée (Rolston et al, 1984 ; Grundmann et Rolston, 1987). Hansen et al (1991) ont utilisé la fonction proposée par Rolston et al (1984) pour modéliser la dénitrification à une échelle millimétrique. Lafolie (1991) a proposé une fonction au regard des travaux de Rolston et al (1984) et de Johnsson et al (1987) dans le même but. Ce faisant, ces auteurs ont supposé que la relation entre la teneur en eau moyenne et la dénitrification globale était identique à la relation entre la teneur en eau locale et la dénitrification locale. Une telle hypothèse ne peut pas être acceptée, 
car il existe des variations spatiales de la teneur en eau et de l'activité microbienne. De plus, la concentration en $\mathrm{O}_{2}$ moyenne peut changer avec la profondeur, et alors la relation entre la fraction anaérobie d'un sol et la teneur en eau doit dépendre de la profondeur dans le sol. Pour tenir compte des variations spatiales de la teneur en $\mathrm{O}_{2}$, Rijtema et Kroes (1991) et Refsgaard et al (1991) ont introduit le transport de $\mathrm{I}^{\prime} \mathrm{O}_{2}$ et sa consommation pour décrire l'activité microbienne du sol.

Dans cet article, nous synthétisons un ensemble de travaux théoriques et expérimentaux visant à caractériser le transport de $\mathrm{I}^{\prime} \mathrm{O}_{2}$ dans le sol et, plus spécifiquement, la fraction du sol en condition d'anoxie. Ce travail est basé sur l'étude numérique des transferts d' $\mathrm{O}_{2}$ dans les sols à structure agrégée. Il est complété par une étude expérimentale des transferts au sein des agrégats. En effet, tous les modèles décrivant l'anoxie dans les sols admettent plusieurs hypothèses généralement non vérifiées. Ces hypothèses portent sur l'homogénéité physique et biologique des agrégats et sur la description de la respiration microbienne. Pour ce faire, nous avons étudié la respiration globale des agrégats au moyen de mesures chromatographiques et de mesures polarographiques (influence de la concentration en $\mathrm{O}_{2}$, inhibition par le $\mathrm{CO}_{2}$, variabilité interagrégats). Le coefficient de diffusion de $\mathrm{I}^{\prime} \mathrm{O}_{2}$ interne à l'agrégat et la distribution de $\mathrm{I}^{\prime} \mathrm{O}_{2}$ au sein des agrégats ont été étudiés par l'utilisation de microélectrodes à $\mathrm{O}_{2}$.

\section{MODÉLISATION DES TRANSFERTS D'OXYGĖNE}

\section{Organisation du sol}

Le sol est assimilé à un ensemble d'agrégats de forme et de dimension variées. $V_{t}$ étant le volume d'un agrégat et $S_{t}$ sa surface externe, il est possible de définir un rayon $r_{e}$ par analogie avec les caractéristiques d'une sphère:

$$
r_{e}=\frac{3 V_{t}}{S_{t}}
$$

Nous admettons que la distribution volumique des rayons $r_{e}$ est de type log-normal. Les agrégats sont en contact avec leurs voisins. Au contraire des travaux précédents, ces contacts sont supposés non négligeables. Nous faisons l'hypothèse que les zones de contact entre agrégats sont isolantes pour les transferts d' $\mathrm{O}_{2}$. L'espace poral est divisé en un espace poral intra-agrégat et un espace poral interagrégats. L'eau sature d'abord l'espace poral intra-agrégat et peut, ultérieurement, envahir aussi l'espace poral interagrégats. Dans l'espace poral interagrégats, l'eau apparaît d'abord en ménisques autour des agrégats (Leffelaar, 1977 ; Leffelaar, 1979). Cette position décroît encore la surface où les transferts d' $\mathrm{O}_{2}$ sont possibles.

\section{Modélisation des transferts 3-D dans des agrégats de formes et de tailles variées}

La distribution des concentrations en $\mathrm{O}_{2}$ dans l'espace et le temps est donnée par la solution de l'équation tridimensionelle suivante :

$$
\hat{\varepsilon}(\omega) \frac{\partial C_{O_{2}}}{\partial t}=\operatorname{div}\left(\left[D_{a g}(\omega)\right] \vec{\nabla} C_{O_{2}}\right)-R_{O_{2}}(\omega)
$$

où $\omega$ est le vecteur donnant la position au sein de l'agrégat $(\mathrm{m}, \mathrm{m}, \mathrm{m}), \mathrm{C}_{\mathrm{O}_{2}}$ est la concentration en $\mathrm{O}_{2}$ transformée en sa concentration en équilibre dans l'air ( $\mathrm{mol} \mathrm{m}^{-3}$ ), $\mathrm{t}$ est la date (s), $\hat{\varepsilon}$ est la porosité équivalente, $D_{a g}$ est le coefficient de diffusion dans l'agrégat lorsque les concentrations en $\mathrm{O}_{2}$ sont transformées en concentrations en équilibre dans l'air $\left(\mathrm{m}^{2} \mathrm{~s}^{-1}\right), \mathrm{R}_{\mathrm{O}_{2}}$ est la respiration microbienne ( $\mathrm{mol} \mathrm{m}^{-3} \mathrm{~s}^{-1}$ ), $\partial$ est l'opérateur différentiel, div est l'opérateur divergence et $\vec{\nabla}$ est l'opérateur nabla.

$D_{\mathrm{ag}}, \hat{\varepsilon}$ et $\mathrm{R}_{\mathrm{O}_{2}}$ peuvent dépendre de $\omega$ pour tenir compte des hétérogénéités spatiales. En plus, $\mathrm{R}_{\mathrm{O}_{2}}$ dépend de la concentration en $\mathrm{O}_{2}$. Pour des agrégats homogènes, l'équation précédente peut être simplifiée :

$$
\hat{\varepsilon} \frac{\partial C_{O_{2}}}{\partial t}=\operatorname{div}\left(\left[D_{a g}\right] \vec{\nabla} C_{O_{2}}\right)-R_{O_{2}}
$$

Cette équation doit être résolue en tenant compte simultanément de conditions initiales et de conditions aux limites des agrégats. Nous avons considéré des conditions de flux nul pour les régions externes de l'agrégat où celui-ci est en contact avec d'autres agrégats ou recouvert par un film d'eau d'épaisseur non négligeable :

$$
\vec{\nabla} C_{O_{2}} \vec{n} I_{\partial \Omega}=0
$$


où $\vec{n}$ est le vecteur normal à la frontière $\partial \Omega \mathrm{du}$ domaine considéré.

Nous avons choisi des conditions de concentration imposée pour les régions externes de l'agrégat en contact avec l'espace poral interagrégats :

$$
\left.C_{\mathrm{O}_{2}}\right|_{\partial \Omega}=C_{i a}
$$

où $\mathrm{C}_{\mathrm{ia}}$ est la concentration en $\mathrm{O}_{2}$ dans l'espace poral interagrégats au niveau de l'agrégat.

À partir de ces équations et des conditions aux limites des agrégats, nous avons réalisé des simulations numériques pour étudier la durée de la phase d'installation des concentrations au sein des agrégats (durée du régime transitoire) pour 2 types de conditions initiales:

$$
\begin{gathered}
C_{O_{2}}(\omega)=0 \\
C_{O_{2}}(\omega)=C_{i a}
\end{gathered}
$$

Pour des agrégats saturés en eau, nous montrons que cette durée n'excède pas $6 \mathrm{~h}$ dans le cas d'agrégats dont la concentration initiale est en équilibre avec celle de l'air et $3 \mathrm{~h}$ dans le cas d'agrégats initialement en état d'anaérobiose totale. Aussi, nous avons privilégié le déterminisme de l'anoxie après que l'équilibre des concentrations en $\mathrm{O}_{2}$ a été atteint.

Pour arriver à ces résultats et aux résultats qui suivent, nous avons résolu l'équation [3] au moyen de la méthode des éléments finis (variante de Galerkin). Cette résolution nous permet d'arriver à la distribution spatiale des concentrations en $\mathrm{O}_{2}$ au sein d'agrégats de forme quelconque et à différentes dates. À partir de ces données, il nous est alors possible de calculer la fraction anoxique de l'agrégat en question.

Pour des agrégats sphériques, Currie (1961) et Greenwood et Berry (1962) ont montré qu'il n'y a pas d'anaérobiose dans un agrégat isolé tant que son rayon est inférieur à un rayon critique $r_{c}$ vérifiant l'équation suivante :

$$
r_{c}=\left(\frac{6 D_{a g} C_{i a}}{M}\right)^{1 / 2}
$$

où $M$ est la respiration microbienne supposée constante tant qu'il y a de 1 ' $\mathrm{O}_{2}$ dans le milieu. Pour des agrégats $r$ de rayon supérieur à $r_{c}$, ils montrent qu'il existe un centre anoxique de rayon $r_{0}$ tel que :

$$
\frac{6 D_{a g} C_{i a}}{M}=r^{2}+2 \frac{r_{0}{ }^{3}}{r}-3 r_{0}^{2}
$$

$r_{0} / r$ est donné par la relation (Smith, 1980) :

$$
\frac{r_{0}}{r}=\frac{-\cos (\theta)}{2 \cos (\theta / 3)}
$$

avec :

$$
\theta=\pi-\sin ^{-1}\left(r_{\mathrm{c}} / r\right)
$$

En considérant $\mathrm{K}$ comme la respiration globale de l'agrégat exprimée par unité de volume d'agrégat incluant les zones anoxiques ne respirant pas, on a alors la relation :

$$
\frac{K}{M}=\frac{r^{3}-r_{0}^{3}}{r^{3}}
$$

où $\mathrm{K} / \mathrm{M}$ est le rapport des volumes respirant et total.

Greenwood et Berry (1962) avaient proposé d'étendre ces résultats à des agrégats de formes diverses et caractérisés par le rapport de leur surface à leur volume. Quelques essais expérimentaux réalisés sur des agrégats artificiels isolés en Agar-Agar montraient que l'extension d'une telle relation semblait possible. Nous avons testé ces mêmes possibilités d'extension au moyen de simulations pour des agrégats de formes variées et ayant une fraction de leur surface externe bloquée. Pour ce faire, nous avons défini, par analogie avec les caractéristiques d'une sphère, un rayon équivalent $r$ ' par la relation suivante :

$$
r^{\prime}=\frac{3 V_{t}}{S_{t}-\left(S_{c}+S_{w}\right)}
$$

où $S_{c}$ est la surface de l'agrégat en contact avec d'autres agrégats $\left(\mathrm{m}^{2}\right)$ et $S_{w}$ sa surface couverte par un film d'eau $\left(\mathrm{m}^{2}\right)$.

Dans le cas d'agrégats isolés, il y a un très bon accord entre le modèle sphérique et les résultats que l'on obtient par simulation numérique à partir de la résolution de l'équation (3) pour des agrégats de forme parallélipédique, cylindrique et sphérique (fig 1). Les caractéristiques géométriques des différents agrégats sont données au tableau I. 


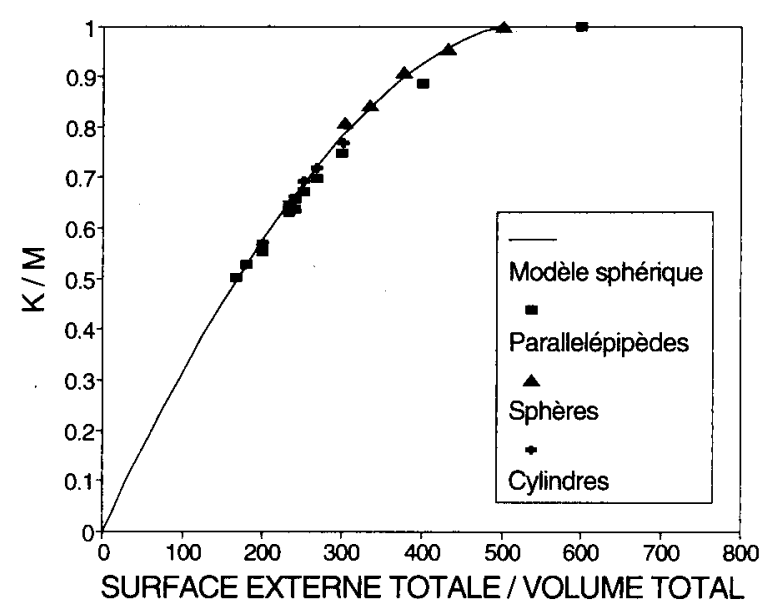

Fig 1. Fraction aérobie à l'équilibre d'agrégats isolés de formes variées avec $D_{a}=10^{-11} \mathrm{~m}^{2} \mathrm{~s}^{-1}$ et $\mathrm{M}=1,5 \mathrm{~mol} \mathrm{~m}-3$ $\mathrm{s}^{-1}$.

Dans le cas d'agrégats ayant une fraction de leur surface externe bloquée, il y a encore un bon accord entre le modèle sphérique et les simulations pour des agrégats ayant un rayon équivalent, en absence de blocages, supérieur ou égal en ordre de grandeur au rayon critique $r_{c}$ malgré une grande variabilité des résultats (fig 2). Cette

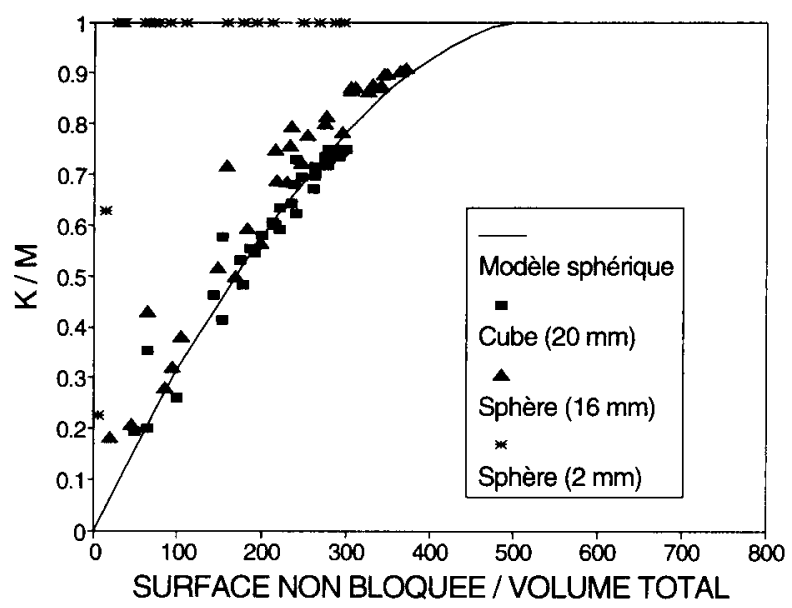

Fig 2. Fraction aérobie à l'équilibre d'agrégats avec blocage d'une partie de leur surface externe avec $D_{a}=10^{-11} \mathrm{~m}^{2} \mathrm{~s}^{-1}$ et $M=1,5 \mathrm{~mol} \mathrm{~m}^{-3}$.

variabilité pourrait s'expliquer par la distribution des surfaces bloquées à la périphérie de l'agrégat. Pour des agrégats très petits par rapport au rayon critique, le modèle sphérique associé au rayon équivalent $r$ ' aboutit à une surévaluation de l'anoxie qui peut être très importante (fig 2). Pour retrouver des estimations correctes, nous

Tableau I. Dimensions des agrégats utilisés pour les simulations (cm).

\begin{tabular}{|c|c|c|c|c|c|c|}
\hline \multirow{2}{*}{$\begin{array}{r}\text { Numéro } \\
\text { d'agrégat }\end{array}$} & \multicolumn{3}{|c|}{ Parallélépipède } & \multicolumn{2}{|c|}{ Cylindre } & \multirow{2}{*}{$\begin{array}{l}\text { Sphère } \\
\text { Rayon }\end{array}$} \\
\hline & Longueur & Largeur & Hauteur & Rayon & Hauteur & \\
\hline 1 & 1,0 & 1,0 & 1,0 & & & \\
\hline 2 & 1,5 & 1,5 & 1,5 & & & \\
\hline 3 & 2,0 & 2,0 & 2,0 & & & \\
\hline 4 & 2,5 & 2,5 & 2,5 & & & \\
\hline 5 & 3,0 & 3,0 & 3,0 & & & \\
\hline 6 & 2,0 & 2,0 & 3,0 & & & \\
\hline 7 & 2,0 & 2,0 & 4,0 & & & \\
\hline 8 & 2,0 & 2,0 & 5,0 & & & \\
\hline 9 & 2,0 & 2,0 & 6,0 & & & \\
\hline 10 & 2,0 & 2,0 & 6,0 & & & \\
\hline 11 & 2,0 & 4,0 & 4,0 & & & \\
\hline 12 & 2,0 & 5,0 & 5,0 & & & \\
\hline 13 & 2,0 & 6,0 & 6,0 & & & \\
\hline 14 & & & & 1,0 & 2,0 & \\
\hline 15 & & & & 1,0 & 3,0 & \\
\hline 16 & & & & 1,0 & 4,0 & \\
\hline 17 & & & & 1,5 & 2,0 & \\
\hline 18 & & & & & & 0,6 \\
\hline 19 & & & & & & 0,7 \\
\hline 20 & & & & & & 0,8 \\
\hline 21 & & & & & & 0,9 \\
\hline 22 & & & & & & 1,0 \\
\hline
\end{tabular}


sommes amenés à proposer de remplacer le rayon critique $r_{C}$ par un rayon critique plus grand après ajustement aux données simulées :

$$
r_{c}^{\prime}=0,067 r_{c}\left[\exp \left(r_{c} / r\right)-\exp (1)\right]+r_{c}
$$

L'anoxie au sein de l'agrégat peut alors encore être estimée en utilisant les équations [9], [10] et [11]. Les détails sur les calculs non présentés ici sont indiqués dans Renault et Stengel (1994).

\section{Modélisation des transferts d'O $\mathrm{O}_{2}$ dans le sol}

La distribution des concentrations en $\mathrm{O}_{2}$ dans l'espace poral interagrégats est donnée par la solution à l'équation suivante :

$$
\varepsilon_{i a}(z) \frac{\partial C_{O_{2}}}{\partial t}=\frac{\partial}{\partial z}\left(D_{i a}(z) \frac{\partial C_{O_{2}}}{\partial z}\right)-K^{\prime}(z)[14]
$$

où $\varepsilon_{\mathrm{ia}}$ est la porosité interagrégats libre à l'air $\left(\mathrm{m}^{3} \mathrm{~m}^{-3}\right), \mathrm{D}_{\mathrm{ia}}$ est le coefficient de diffusion interagrégats $\left(\mathrm{m}^{2} \mathrm{~s}^{-1}\right)$ et $\mathrm{K}^{\prime}(\mathrm{z})$ correspond à la respiration globale des agrégats $(K(z))$ rapportée non plus au volume de l'agrégat mais au volume de sol.

L'équation [14] a été résolue au moyen de la méthode des éléments finis (variante de Galerkin). Cette résolution nous permet d'arriver à la distribution spatiale des concentrations en $\mathrm{O}_{2}$ en un point quelconque de l'espace poral interagrégat et à différentes dates. À partir de ces données, il nous est alors possible de calculer la fraction anoxique des agrégats à toutes les profondeurs.

$K^{\prime}(z)$ dépend de la concentration en $\mathrm{O}_{2}$ dans l'espace poral interagrégat, donc de la profondeur $z$. Son estimation nécessite de connaître l'importance des surfaces de contact entre agrégats et leur recouvrement par de l'eau. Les surfaces de contact ont été estimées par la formule suivante (Renault et Sierra, 1994) :

$$
S_{c} / S_{t}=\left(0,43-\varepsilon_{i a}\right) / 0,43
$$

Les surfaces couvertes par de l'eau ont été estimées par la formule (Renault et Sierra, 1994) :

$$
\frac{S_{w}}{S_{t}-S_{c}}=\left(\frac{\varepsilon_{\mathrm{ia} . \mathrm{w}}}{\varepsilon_{\mathrm{ia}}}\right)^{0,5}
$$

où $\varepsilon_{\text {ia.w }}$ est la porosité interagrégats occupée par de l'eau.
Ainsi, pour un profil de sol de $0,25 \mathrm{~m}$ de profondeur (on suppose qu'à $0,25 \mathrm{~m}$ il n'y a plus de flux d' $^{\prime} \mathrm{O}_{2}$ vers la profondeur), on montre que la prise en compte des contacts entre agrégats et de leur recouvrement par de l'eau a une influence considérable sur les résultats (fig $3 a$ et $b$ ). $\mathrm{Si}$ l'on néglige ces contacts et le recouvrement des agrégats par de l'eau, la respiration globale des agrégats est plus grande pour une concentration en $\mathrm{O}_{2}$ donnée dans l'espace poral interagrégats d'après nos simulations numériques. Par voie de conséquence, l'anoxie au sein des agrégats est plus faible en surface, mais elle peut devenir facilement généralisée au-delà d'une profondeur donnée. On montre par ailleurs l'existence d'un effet de la structure du sol qui s'exerce principalement par l'intermédiaire de la distribution granulométrique des agrégats (fig 4). On montre enfin l'effet de la température (fig $5 a$ et b). Cet effet dépend fortement de la teneur en eau : il est négligeable à saturation (puisque tout le volume du sol est en état d'anoxie) et maximal lorsque
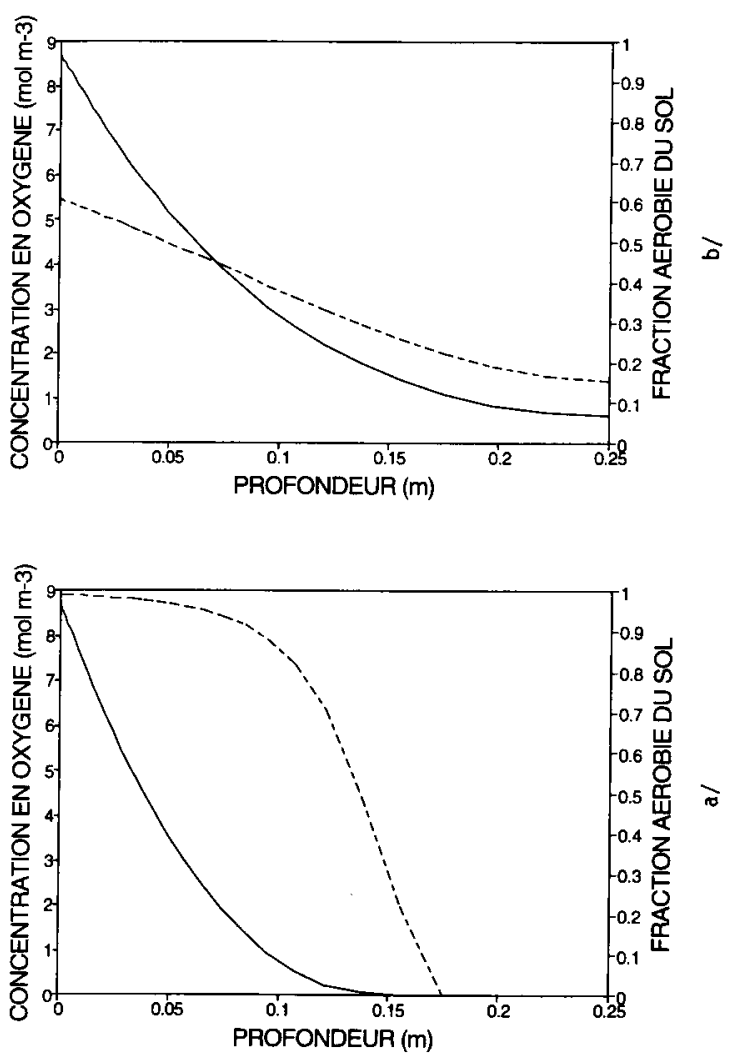

Fig 3. Concentration en $\mathrm{O}_{2}$ dans l'espace poral interagrégat $(-)$ et fraction aérobique des agrégats (----) en fonction de la profondeur à $\mathrm{T}=293^{\circ} \mathrm{K}$ pour un sol ayant une densité apparente de 1,1 , un rayon moyen de l'agrégat de $0,0025 \mathrm{~m}$ et un taux de saturation en eau de 0,932. a. La surface externe disponible pour les échanges $\mathrm{d}^{\prime} \mathrm{O}_{2}$ entre l'agrégat et l'espace poral interagrégat est égale à la totalité de la surface externe de l'agrégat. b. La surface externe disponible pour les échanges d' $\mathrm{O}_{2}$ entre l'agrégat et l'espace poral interagrégat est égale à la surface externe de l'agrégat qui n'est pas en contact avec d'autres agrégats et qui n'est pas recouverte par de l'eau. 
l'eau sature les agrégats mais est absente de l'espace poral interagrégats. Cet effet température peut entraîner l'existence de $Q_{10}$ apparents pour la dénitrification très supérieurs à 2 . En effet, le $Q_{10}$ combine les effets de la température sur le volume des sites anoxiques et les effets de

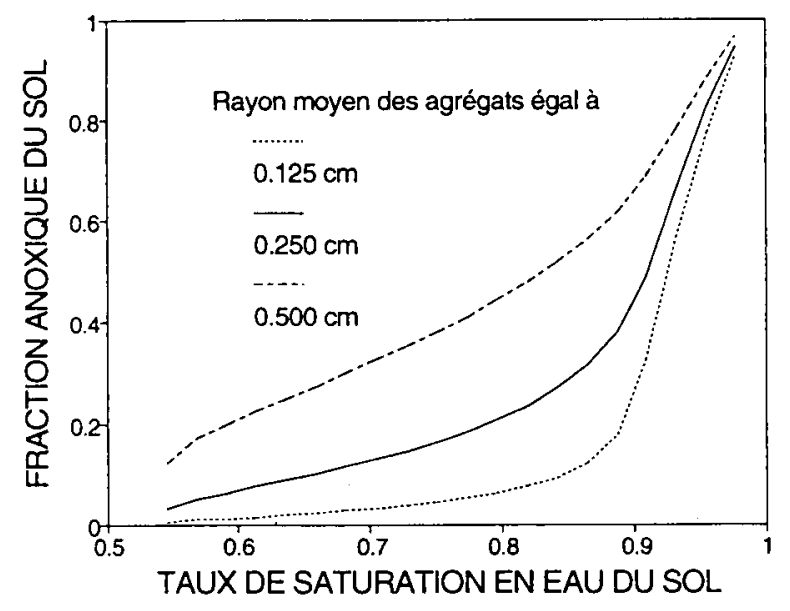

Fig 4. Influence de la distribution du rayon des agrégats sur leur fraction anoxique à $T=293 \mathrm{~K}$ pour un sol de densité apparente de 1,1 .
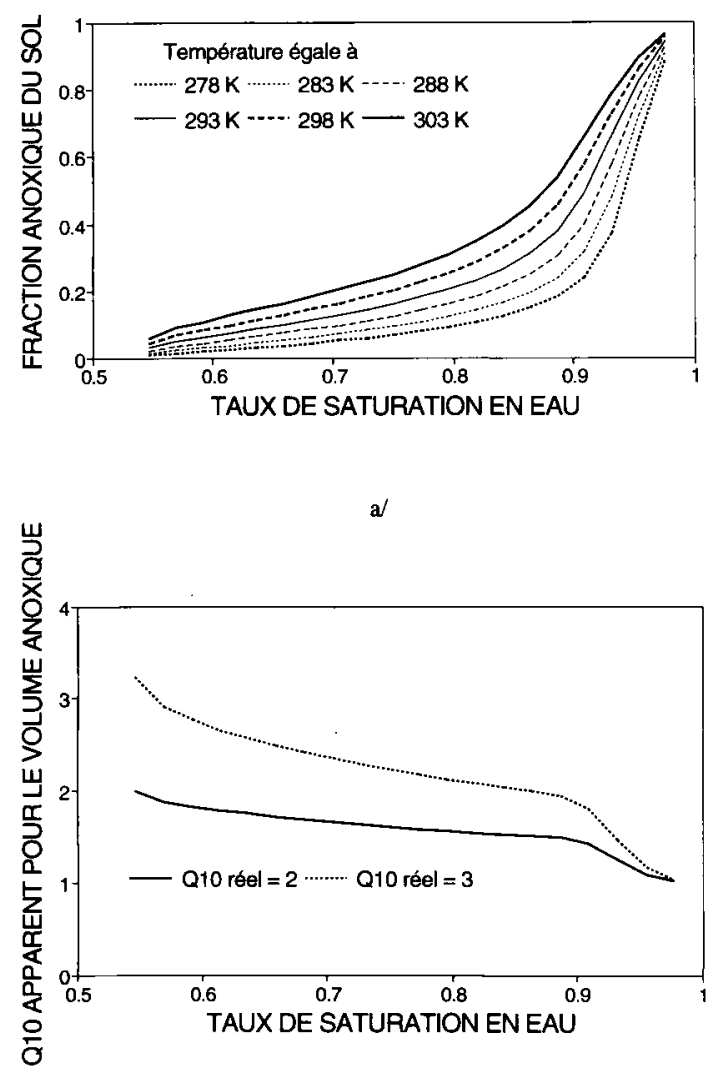

b/

Fig 5. Influence de la température sur la fraction anoxique des agrégats pour un sol ayant une densité apparente de 1,1 et un rayon moyen d'agrégats de 0,002 $5 \mathrm{~m}$. a. Relation entre le taux de saturation en eau et la fraction anoxique des agrégats pour un $\mathrm{Q}_{10}$ réel de 2 pour la consommation en $\mathrm{O}_{2}$. b. $Q_{10}$ apparent autour de $293 \mathrm{~K}$ pour la fraction anoxique des agrégats en fonction du taux de saturation en eau du sol la température sur l'activité microbienne (dont l'activité dénitrifiante) dans ces sites. Ces résultats sont en accord avec les résultats expérimentaux de Craswell (1978). Enfin, les résultats que nous avons obtenus sont similaires aux relations entre la dénitrification et la teneur en eau (fig 6). Cela conforte l'idée que la teneur en eau a un effet sur la dénitrification essentiellement par le biais du volume anoxique. Cette conclusion est conforme aux résultats expérimentaux de Sexstone et al (1988) sur la dénitrification.

\section{MATÉRIELS ET MÉTHODES EXPÉRIMENTALES}

\section{Le sol}

Nous avons travaillé sur un sol limono-sableux prélevé dans l'est de la France à environ $20 \mathrm{~km}$ de Dijon et utilisé par Hénault (1993) pour une étude sur la dénitrification. Le sol présente des traces d'hydromorphie temporaires ou passées (présence de concrétions ferro-manganiques). II est caractérisé par une dénitrification habituellement importante (JC Germon, communication personnelle). Des agrégats ont été prélevés dans la couche travaillée entre 10 et $25 \mathrm{~cm}$ de profondeur afin de minimiser l'importance des racines d'un blé en début de montaison. Les caractéristiques générales de cette couche de sol sont reportées dans le tableau II. Ces agrégats ont été tamisés et fractionnés en 12 classes granulométriques (tableau III). Pour obtenir des quantités similaires d'agrégats des différentes tailles, les mottes ont souvent été fractionnées manuellement. Les analyses faites séparément sur chaque classe granulométrique montrent qu'il n'y

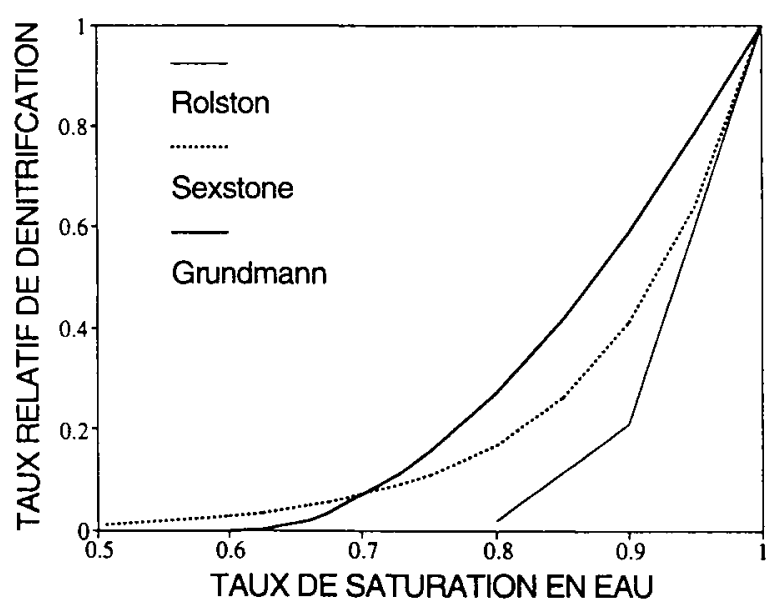

Fig 6. Taux relatif de dénitrification en fonction du taux de saturation en eau du sol. Les relations proposées sont issues des données expérimentales. 
Tableau II. Principales caractéristiques du sol utilisé.

\begin{tabular}{|c|c|}
\hline \multicolumn{2}{|c|}{ Composition granulométrique } \\
\hline Argile (\%) & 14,9 \\
\hline Limon fin $(\%)$ & 24,8 \\
\hline Limon grossier (\%) & 27,4 \\
\hline Sable fin (\%) & 22,9 \\
\hline Sable grossier (\%) & 10,0 \\
\hline \multicolumn{2}{|c|}{ Données de l'analyse chimique } \\
\hline Matières organiques (\%) & 1,62 \\
\hline Carbone organique (\%) & 0,81 \\
\hline Azote organique (\%) & 0,085 \\
\hline Rapport C/N & 9,5 \\
\hline $\mathrm{N}-\mathrm{NO}_{3}(\mathrm{ppm})$ & 0,25 \\
\hline $\mathrm{N}-\mathrm{NH}_{4}(\mathrm{ppm})$ & 5,75 \\
\hline pH eau & 7,8 \\
\hline $\mathrm{pH} \mathrm{KCl} 1 \mathrm{~N}$ & 7,0 \\
\hline $\mathrm{CEC}(\mathrm{meq} \%)$ & 7,6 \\
\hline $\mathrm{CaCO}_{3}$ total $(\%)$ & Traces \\
\hline Mn échang (ppm) & 1,3 \\
\hline Fe libre (\%) & 1,03 \\
\hline
\end{tabular}

Tableau III. Classes d'agrégats retenues.

\begin{tabular}{rc}
\hline Classe & Taille $(\mathrm{mm})$ \\
\hline 1 & $25-30$ \\
2 & $20-25$ \\
3 & $15-20$ \\
4 & $12-15$ \\
5 & $10-12$ \\
6 & $8-10$ \\
7 & $6-8$ \\
8 & $5-6$ \\
9 & $4-5$ \\
10 & $3-4$ \\
11 & $2-3$ \\
12 & $1-2$ \\
\hline
\end{tabular}

a pas de corrélation entre la taille de la motte et les résultats de ces analyses (matières organiques totales, matières organiques légères, densité apparente).

Parallèlement, des agrégats de sol remaniés ont été fabriqués à partir du même sol selon un protocole précédemment décrit (Fies et Stengel, 1981). De la terre fine a été humidifiée en présence d'un excès d'eau ( 1,9 fois l'humidité équivalente du sol) afin d'obtenir une pâte homogène. Cette pâte a été desséchée très lentement dans le but d'éviter l'apparition de fissures. Les massifs secs obtenus ont alors été découpés en cubes de dimensions variées et usinés dans une éplucheuse à pomme de terre afin de leur confé- rer une forme sphérique. Les agrégats ainsi obtenus ont été ultérieurement réhumectés puis conservés 2 sem ou plus à la température du laboratoire avant expérimentation. De façon générale, nous avons travaillé sur des agrégats théoriquement saturés, à l'exception des expérimentations réalisées sur des agrégats de sol remanié pour l'étude de la respiration microbienne et de son inhibition par le $\mathrm{CO}_{2}$. Pour ces expérimentations, le taux de saturation recherché était d'environ $90 \%$ afin d'éviter l'existence de gradients de concentration de $\mathrm{O}_{2}$ et/ou de $\mathrm{CO}_{2}$ au sein des agrégats.

\section{Distribution des concentrations en $\mathrm{O}_{2}$ et coefficient de diffusion au sein des agrégats}

Des micro-électrodes à $\mathrm{O}_{2}$ ont été fabriquées selon un protocole proposé initialement par Revbech et Ward (1983), largement décrit par Revsbech et Jorgensen (1986), et ultérieurement amélioré par Revsbech (1989). Une description générale de l'électrode est proposée sur la figure 7. Pratiquement, on mesure un courant électrique (dont l'ordre de grandeur est de $100 \mathrm{pA}$ ) directement lié à la réduction de l' $\mathrm{O}_{2}$ au niveau d'une cathode en or. Lorsque la différence de potentiel entre cette cathode et une électrode de référence est fixée à $0,75 \mathrm{~V}$ environ, $\mathrm{I}_{2} \mathrm{O}_{2}$ est tota-
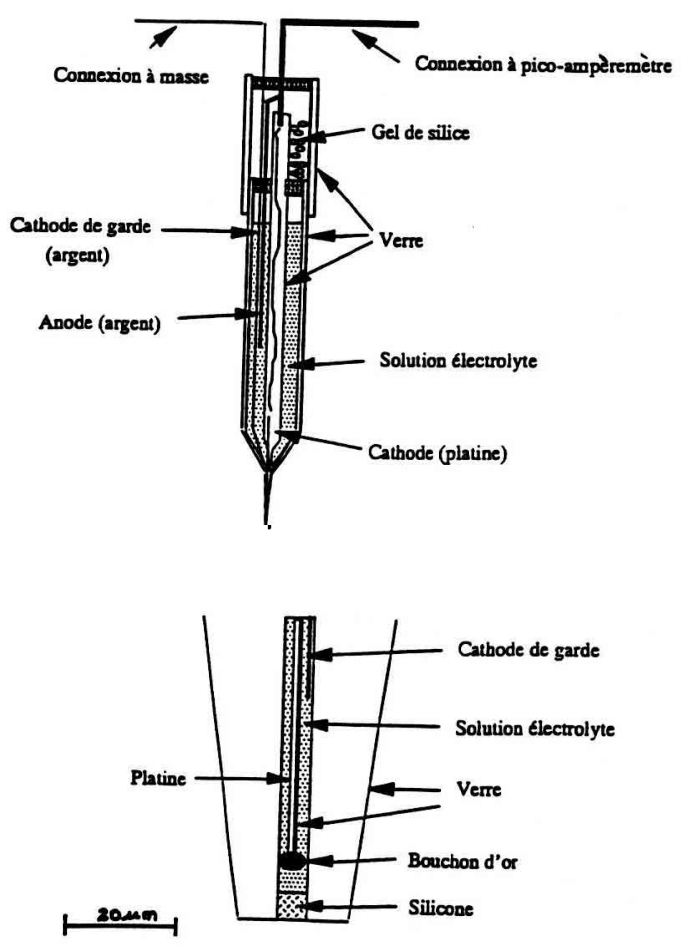

Fig 7. Description de la micro-électrode à $\mathrm{O}_{2}$. a. Schéma général. b. Description détaillée de la pointe de l'électrode. 
lement réduit au niveau de la cathode et le courant électrique, proportionnel au flux d' $\mathrm{O}_{2}$ entre la pointe de l'électrode et la cathode en or, devient proportionnel à la concentration externe en $\mathrm{O}_{2}$. En fait, un courant parasite peut exister. Il est lié à la migration d'ions au sein de la solution et à d'autres processus mal maîtrisés. La mise en place d'une électrode «de garde» en argent et au même potentiel que la cathode en or permet de réduire les champs électriques entre cette cathode et la solution d'électrolyte de l'électrode. Le bruit résiduel peut ainsi être réduit à quelques $\mathrm{pA}$. Une telle électrode peut mesurer des concentrations en $\mathrm{O}_{2}$ dans l'air, dans l'eau ou dans un milieu poreux. Sa consommation en $\mathrm{O}_{2}$ est négligeable et ne provoque aucune modification des concentrations mesurées. La taille de la pointe (entre $10 \mu \mathrm{m}$ et $50 \mu \mathrm{m})$ ne provoque pas de perturbation du signal lorsque l'électrode est insérée dans un milieu poreux saturé. Ces électrodes ont été utilisées pour estimer le coefficient de diffusion intra-agrégat et pour obtenir des distributions $d^{\prime} \mathrm{O}_{2}$ au sein des agrégats.

L'estimation du coefficient de diffusion a été réalisée sur une série d'agrégats naturels et artificiels après stérilisation par irradiation afin d'éliminer toute consommation interne d' $\mathrm{O}_{2}$. Pour ce faire, l'électrode était placée à une profondeur fixée au sein de l'agrégat. La concentration externe en $\mathrm{O}_{2}$ était brutalement modifiée (passage de $21 \%$ à $100 \%$ d' $_{2}$ ) et l'on suivait alors l'évolution de la concentration en $\mathrm{O}_{2}$ au niveau de la pointe de l'électrode. À partir d'un modèle décrivant le transfert de $\mathrm{I}^{\prime} \mathrm{O}_{2}$ dans une sphère homogène (équation [3] en coordonnées sphériques et en absence de respiration microbienne), on estimait alors un coefficient de diffusion par ajustement des données simulées aux données expérimentales. La même opération était répétée à 3 ou 4 profondeurs afin de tester la méthode et l'homogénéité des agrégats analysés.

Des profils d' $\mathrm{O}_{2}$ ont été mesurés pour une concentration externe en $\mathrm{O}_{2}$ égale à $21 \%$. Les mesures ont été réalisées sur des agrégats naturels et remaniés de différentes tailles. Elles ont été effectuées à l'aide d'un micro-manipulateur pour des déplacements de 0,1 à $0,25 \mathrm{~mm}$ entre 2 points de mesure successifs.

Sexstone et al (1985) avaient déjà utilisé des micro-électrodes à $\mathrm{O}_{2}$ à des fins similaires. Leurs électrodes ne comportaient pas de cathode de garde et les mesures de diffusion qu'ils réalisaient se faisaient sur des agrégats non stérilisés.

\section{Respiration globale des agrégats et effet de l' $\mathrm{O}_{2}$ et du $\mathrm{CO}_{2}$}

On a mesuré la respiration globale d'agrégats naturels de différentes tailles (par lots) pour différentes concentrations en $\mathrm{O}_{2}$. Ces mesures ont été réalisées à l'aide des micro-électrodes à $\mathrm{O}_{2}$ précédemment décrites sans fixation du $\mathrm{CO}_{2}$ produit par la respiration. Les expérimentations se faisaient sur des durées de $5 \mathrm{j}$. La respiration a été estimée à partir du changement de la concentration en $\mathrm{O}_{2}$ à l'intérieur des enceintes utilisées pour le confinement des agrégats. La respiration globale des agrégats a par ailleurs été estimée individuellement sur 62 agrégats de 4 $\mathrm{mm}$ de rayon environ.

Pour l'estimation de la respiration globale des agrégats artificiels et pour l'appréhension des effets de $\mathrm{lO}_{2}$ et du $\mathrm{CO}_{2}$, un chromatographe en phase gazeuse a été utilisé afin de mesurer simultanément les concentrations des 2 gaz. Les concentrations en $\mathrm{O}_{2}$ ont varié de $2 \%$ à $21 \%$ et celles du $\mathrm{CO}_{2}$ de $0 \%$ à $12 \%$. Pour obtenir une concentration de $0 \%$ en $\mathrm{CO}_{2}$, celui produit par la respiration des agrégats était fixé par une solution de potasse placée dans l'enceinte de confinement des agrégats. Les expériences duraient encore $5 \mathrm{j}$. Pratiquement nous n'avons pas pu estimer la production de $\mathrm{CO}_{2}$ des agrégats de sol remaniés en raison du fort pouvoir d'absorption du sol lié à son $\mathrm{pH}(7,8)$ (importance de l'ion $\mathrm{HCO}_{3}{ }^{-}$).

\section{RÉSULTATS EXPÉRIMENTAUX}

\section{Estimation du coefficient de diffusion au sein des agrégats}

Deux exemples de variation de la concentration en $\mathrm{O}_{2}$ au cours du temps sont présentés sur les figures $8 a$ et $8 b$. Pour les agrégats de sol remanié, on présente aussi l'ajustement de ces données au moyen d'un modèle décrivant le transfert de $\mathrm{I}^{\prime} \mathrm{O}_{2}$ dans un agrégat sphérique (équation [3] en coordonnées sphériques et en absence de respiration microbienne). L'ordre de grandeur du coefficient de diffusion ajusté $\left(10^{-11} \mathrm{~m}^{2} \mathrm{~s}^{-1}\right)$ est en accord avec les résultats obtenus pour des agrégats naturels par Sexstone et al (1985).

Pour les agrégats de sol remanié, le rapport entre l'expérience et le modèle est très satisfaisant (fig 8a) : l'estimation du coefficient de diffusion ne dépend pas de la position de l'électrode au sein de l'agrégat et les données expérimen- 
tales peuvent être ajustées indifféremment toutes ensembles (un seul ajustement pour l'ensemble des profondeurs observées) ou séparément (un ajustement pour chaque position de la microélectrode). Le coefficient de diffusion estimé pour l'agrégat de la figure $8 a$ est de $1,4910^{-11} \mathrm{~m}^{2} \mathrm{~s}^{-1}$.

Au contraire, il existe un désaccord entre le modèle et l'expérience pour les agrégats naturels. On observe par exemple sur la figure $8 b$ que l'évolution de la concentration en $\mathrm{O}_{2}$ à $2,5 \mathrm{~mm}$ est plus rapide qu'à $1,0 \mathrm{~mm}$ de profondeur pour l'agrégat considéré. Ce résultat peut être interprété par la présence de chemins préférentiels de transfert en surface ou au sein de l'agrégat. De façon plus générale, une hétérogénéité de distribution de l'espace poral avec, éventuellement, des macropores partiellement saturés (galeries de vers, lieux de passage d'une ancienne racine, fissures) va rendre inadéquate l'utilisation d'un modèle de transfert en milieu homogène. Le coefficient de diffusion estimé pour les 2 premières profondeurs $\left(1,3710^{-11} \mathrm{~m}^{2} \mathrm{~s}^{-1}\right.$ à $0,5 \mathrm{~mm}, 1,40$

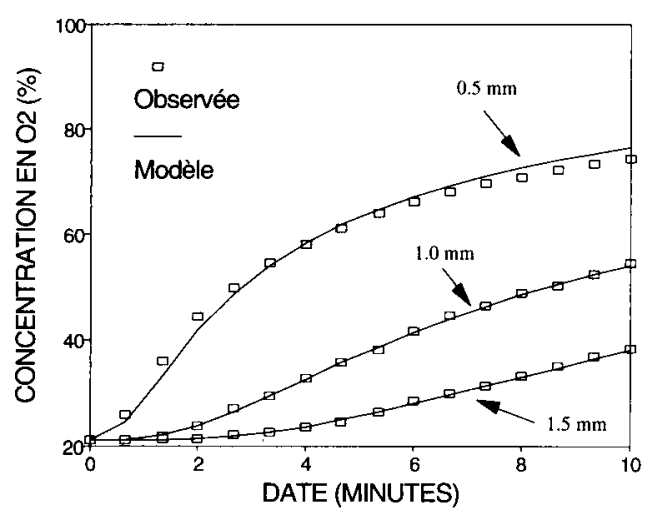

$a^{\prime}$

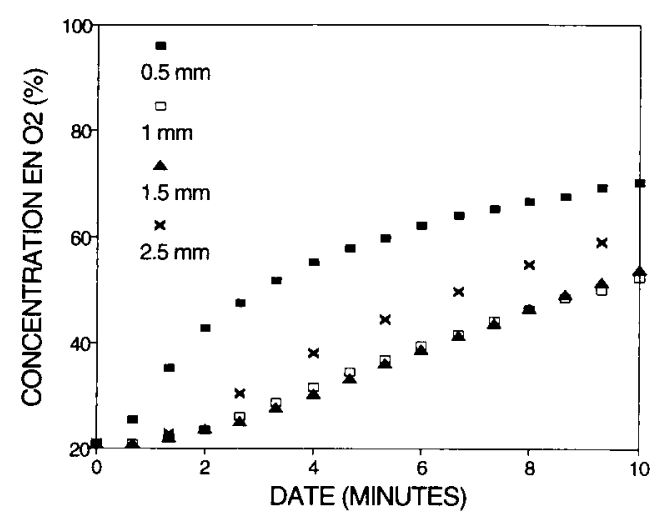

b/

Fig 8. Variations au cours du temps de la concentration en $\mathrm{O}_{2}$ à différentes profondeurs dans un agrégat après passage brutal de la concentration en $\mathrm{O}_{2}$ à sa périphérie de $21 \%$ à $100 \%$. a. Agrégat de sol remanié. b. Agrégat naturel.
$10^{-11} \mathrm{~m}^{2} \mathrm{~s}^{-1}$ à $1,0 \mathrm{~mm}$, et $1,3910^{-11} \mathrm{~m}^{2} \mathrm{~s}^{-1}$ pour l'ensemble des 2 profondeurs) ne diffère pas significativement des valeurs obtenues pour les agrégats de sol remaniés.

\section{Effet de l'O $\mathrm{O}_{2}$ et du $\mathrm{CO}_{2}$ sur la respiration}

Les résultats de l'expérimentation réalisée sur des agrégats de sol remanié, saturés à $90 \%$ et placés dans différentes ambiances gazeuses, ont montré que l'effet de $\mathrm{l}^{\prime} \mathrm{O}_{2}$ pouvait être décrit, dans la gamme des concentrations étudiées, par la loi de Michaelis Menten. Nous avons combiné à cette loi une inhibition de type compétitif due à la présence éventuelle de $\mathrm{CO}_{2}$ (fig $9 \mathrm{a}$ et $b$ ).

$$
R_{\mathrm{O}_{2}}=R_{\mathrm{O}_{2}-\max } \frac{C_{\mathrm{O}_{2}}}{K_{m-\mathrm{O}_{2}}\left(1+\frac{C_{\mathrm{CO}_{2}}}{K_{i-\mathrm{O}_{2}}}\right)+C_{\mathrm{O}_{2}}}
$$
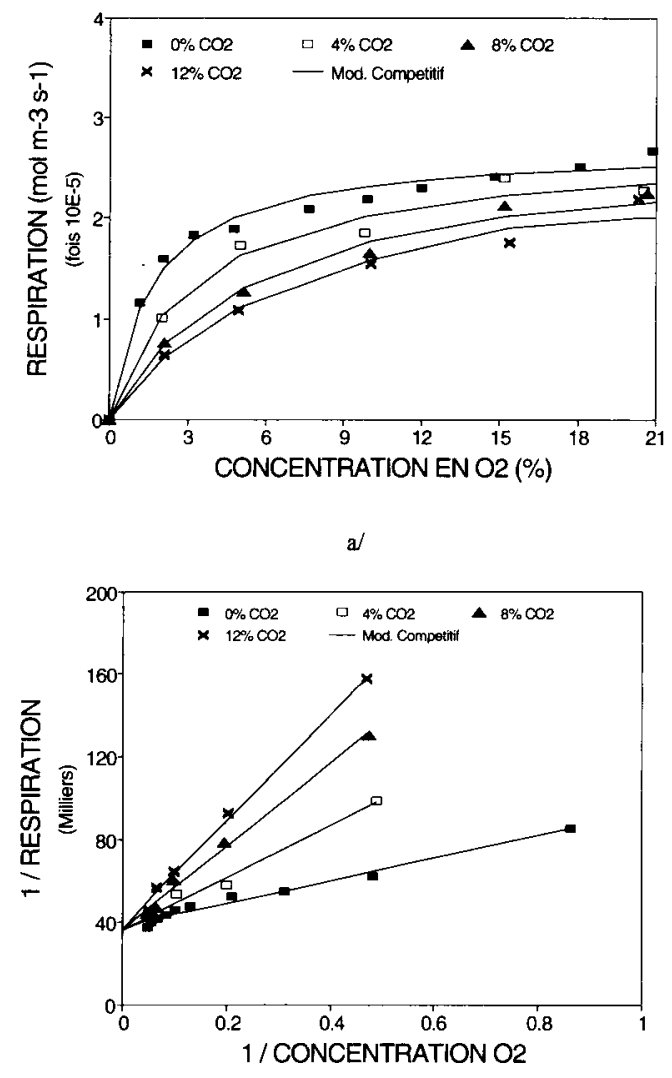

b/

Fig 9. Influence de $\mathrm{I}_{2} \mathrm{O}$ et du $\mathrm{CO}_{2}$ sur la consommation en $\mathrm{O}_{2}$ d'agrégats de sol remaniés saturés en eau à $90 \%$. a. Relation entre la respiration et la concentration en $\mathrm{O}_{2}$ à différentes concentrations en $\mathrm{CO}_{2}$. b. Relation entre l'inverse de la respiration et l'inverse de la concentration en $\mathrm{O}_{2}$ à différentes concentrations en $\mathrm{CO}_{2}$. 
où $R_{\mathrm{O}_{2} \text {-max }}$ est la respiration maximale en absence de limitations liées à l' $\mathrm{O}_{2}$ ou au $\mathrm{CO}_{2}$ $\left(\mathrm{mol} \mathrm{m} \mathrm{m}^{-3} \mathrm{~s}^{-1}\right) ; K_{m-\mathrm{O}_{2}}$ est la constante de Michaelis pour l' $\mathrm{O}_{2}\left(\mathrm{~mol} \mathrm{~m}^{-3}\right)$; et $\mathrm{K}_{\mathrm{i}-\mathrm{O}_{2}}$ est la constante d'inhibition de la consommation d' $\mathrm{O}_{2}$ par le $\mathrm{CO}_{2}\left(\mathrm{~mol} \mathrm{~m}^{-3}\right)$.

Cependant, le mode d'action réel du $\mathrm{CO}_{2}$ reste inconnu. II pourrait être lié à l'influence directe de ce composé sur la respiration microbienne. Mais il pourrait aussi agir indirectement sur celle-ci par l'intermédiaire du $\mathrm{pH}$ du sol qu'il modifie (et des déplacements des équilibres physico-chimiques associés au $\mathrm{pH}$ ) et/ou par son influence éventuelle sur la croissance microbienne. Cela apparaît d'autant plus vraisemblable que les résultats obtenus sur agrégats naturels (Sierra, 1994) sont quelque peu différents. Ainsi, si le $\mathrm{CO}_{2}$ a généralement un effet inhibiteur sur la respiration de ces derniers, il peut l'activer à faible concentration : la respiration croît d'environ $10-15 \%$ lorsque l'on passe de 0 à $4 \%$ de $\mathrm{CO}_{2}$.

À partir de l'expérimentation réalisée sur agrégats de sol remanié, nous estimons la constante de Michaelis Menten pour la consommation d' $\mathrm{O}_{2}$ $\left(\mathrm{K}_{\mathrm{m}-\mathrm{O}_{2}}\right)$ comme égale à $1,6 \%$ d' $\mathrm{O}_{2}$ et la constante d'inhibition associée à cette même consommation $\left(\mathrm{K}_{\mathrm{i}-\mathrm{O}_{2}}\right)$ comme égale à $3,52 \%$ de $\mathrm{CO}_{2}$. $\mathrm{La}$ valeur de $\mathrm{K}_{\mathrm{m}-\mathrm{O}_{2}}$ montre que la respiration semble dépendre de la concentration en oxygène contrairement à ce qui est couramment admis. Cette constante pourrait ne pas être une constante d'affinité si la désaturation des agrégats n'était pas suffisante pour éliminer tous les problèmes de transferts d' $\mathrm{O}_{2}$ jusqu'aux bactéries. $\mathrm{La}$ valeur de $\mathrm{K}_{\mathrm{i}-\mathrm{O}_{2}}$ est très petite : elle reflète l'influence très grande du $\mathrm{CO}_{2}$.

II n'est pas possible de comparer nos résultats à des données bibliographiques en raison de l'absence d'études sur le sujet pour les sols. Dans d'autres domaines, l'effet du $\mathrm{CO}_{2}$ a été largement étudié (respiration des fruits et légumes, germination des semences, respiration de bactéries isolées). Son action est variable.

\section{Évolution de la respiration maximale en fonction du temps}

Sur la figure 10 sont présentés plusieurs profils $\mathrm{d}^{\prime} \mathrm{O}_{2}$ mesurés à différentes dates sur un même agrégat de sol remanié. On constate que le profil change avec le temps et que la profondeur à laquelle la concentration d' $\mathrm{O}_{2}$ devient nulle croît.

Ce changement peut être dû à la diminution progressive de l'activité microbienne au sein de

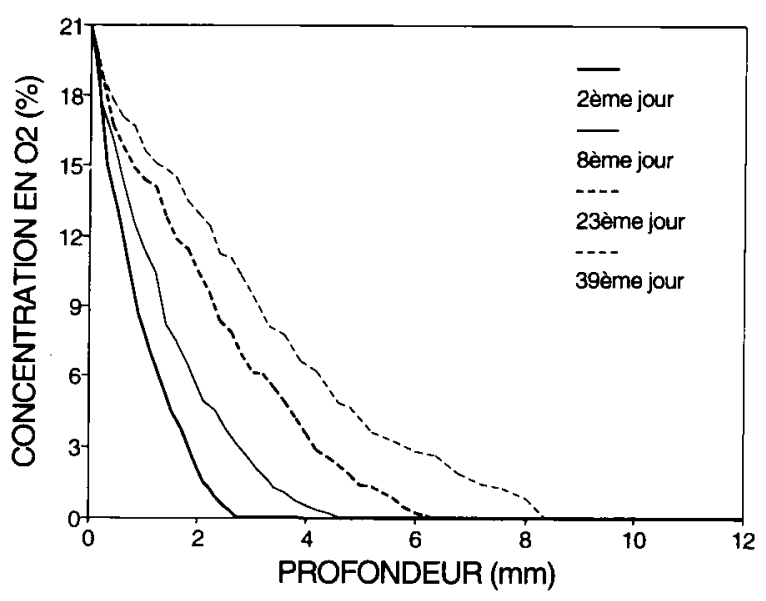

Fig 10. Profils $d^{\prime} \mathrm{O}_{2}$ au sein d'un agrégat en fonction du temps.

l'agrégat. En effet, il est bien connu que la réhumectation d'un sol sec s'accompagne généralement d'une reprise très brutale de l'activité microbienne, celle-ci revenant progressivement à un niveau plus faible. Pour la suite de notre travail, nous avons ignoré d'éventuelles modifications des variables $\mathrm{K}_{\mathrm{m}-\mathrm{O}_{2}}$ et/ou $\mathrm{K}_{\mathrm{i}-\mathrm{O}_{2}}$. Nous pensons que le phénomène prépondérant est la variation de la quantité de carbone facilement métabolisable pour l'activité respiratoire du sol (biomasse microbienne morte avec le dessèchement du sol) qui pourrait changer en l'espace de quelques jours et entraîner une variation de la respiration maximale $\left(\mathrm{R}_{\mathrm{O}_{2}-\max }\right)$.

\section{Respiration globale des agrégats naturels et des agrégats de sol remaniés}

Les résultats des expérimentations relatives à la respiration globale des agrégats naturels et des agrégats de sol remaniés sont présentés aux figures $11 a$ et $b$.

On constate que la respiration des agrégats naturels est supérieure à celle des agrégats de sol remanié. Ce constat est vrai en particulier pour les fortes concentrations en $\mathrm{O}_{2}$ et les plus petites tailles d'agrégats. Dans ce cas, la respiration des agrégats naturels est environ 5 fois plus élevée. L'ordre de grandeur de la respiration des agrégats de sol remanié est par ailleurs plus faible que celui trouvé pour l'étude de l'effet du $\mathrm{CO}_{2}$ sur ces mêmes agrégats. Cet écart peut être lié à l'évolution de la respiration maximale mentionnée dans le paragraphe précédent. En effet, l'influence du $\mathrm{CO}_{2}$ a été étudiée 2 sem après réhumectation des agrégats. Au contraire, l'expé- 


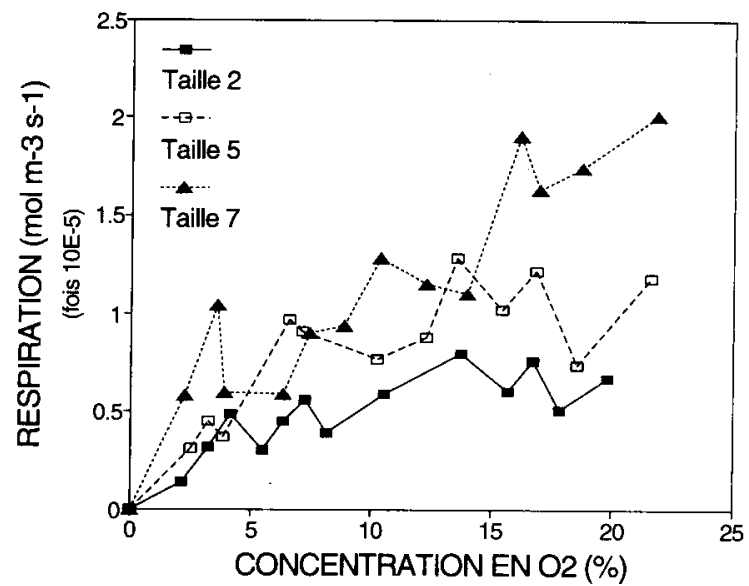

a/

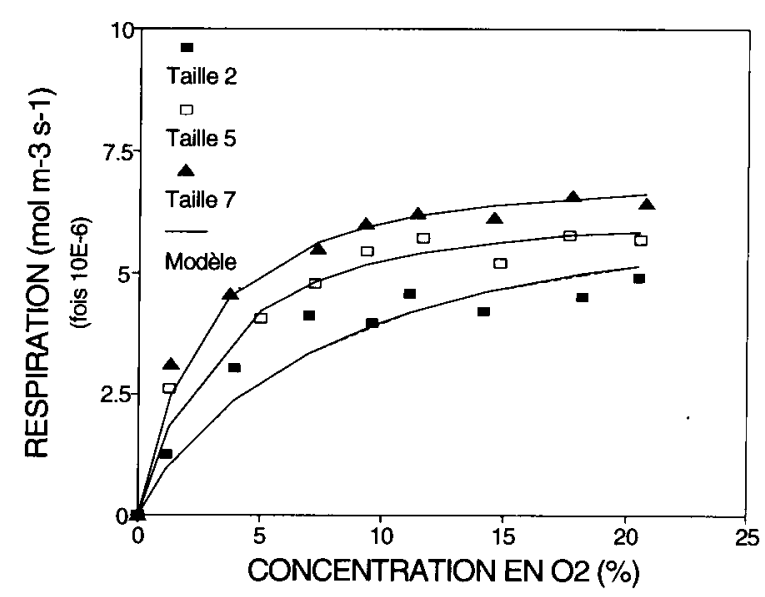

b/

Fig 11. Respiration globale d'agrégats de tailles $2(20-25$ $\mathrm{mm}), 5(10-12 \mathrm{~mm})$ et $7(6-8 \mathrm{~mm})$. a. Agrégats naturels. b. Agrégats de sol remanié.

rimentation présentée ici a été réalisée 2 mois environ après cette même réhumectation.

Par ailleurs, la respiration globale des agrégats naturels présente une grande variabilité. Cette variabilité a été retrouvée au cours de l'expérimentation relative à la respiration de 62 agrégats naturels isolés de rayon proche de $4 \mathrm{~mm}$ (fig 12). La distribution de leur respiration est de type normal et le coefficient de variation de cette respiration est d'environ $45 \%$. II faut noter que, pour cette taille d'agrégats, tout le volume des agrégats est en état d'aérobiose. La variabilité de la respiration globale observée ne peut donc être attribuée à des différences de volume anoxique. Elle peut être due à plusieurs facteurs, mais l'hétérogénéité des transferts gazeux au sein des agrégats et la distribution probablement hétérogène de la matière organique sont sans doute les facteurs dominants.

Nous avons ajusté le modèle (équation [3]) aux données expérimentales après y avoir ajouté

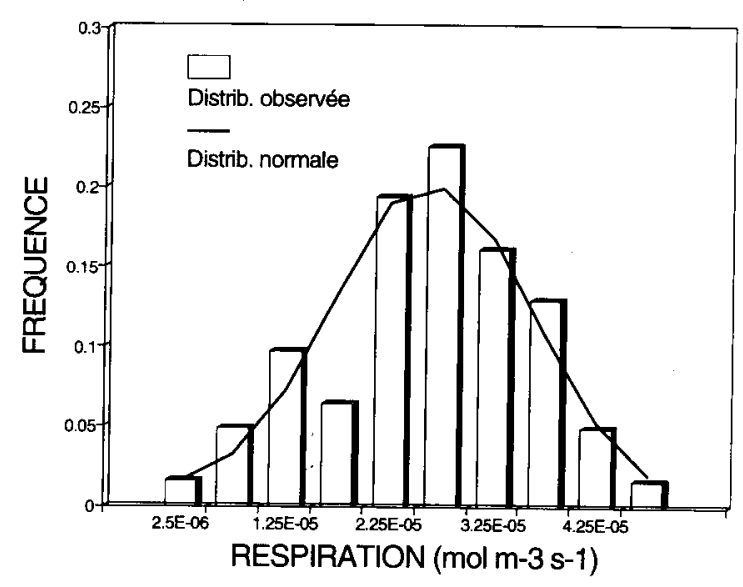

Fig 12. Densité de répartition de la respiration globale des agrégats naturels de rayon proche de $4 \mathrm{~mm}$ (fonction établie à partir de 62 agrégats de sol remanié).

le transfert du $\mathrm{CO}_{2}$, les réactions physico-chimiques qui lui sont associées et son influence sur la respiration (Sierra, 1994). Tous les paramètres du modèle ont été estimés expérimentalement, à l'exception de la respiration maximale $\left(\mathrm{R}_{\mathrm{O}_{2}-\max }\right)$, en raison de sa variation au cours du temps. Pour les agrégats naturels, le modèle décrit au début de cet article ne permet pas d'expliquer a priori les écarts de respiration globale entre tailles d'agrégats aux fortes concentrations en $\mathrm{O}_{2}$ : la respiration globale des agrégats ne semble pas converger vers une valeur indépendante de la taille. Malgré une valeur de $\mathrm{K}_{\mathrm{m}-\mathrm{O}_{2}}$ plus élevée que celles classiquement proposées dans la littérature, l'ajustement des données expérimentales ne peut se faire que séparément pour chaque taille d'agrégat. Pour les agrégats remaniés, l'ajustement du modèle aux données expérimentales peut se faire avec calage pour chaque taille de cette respiration. On constate un bon accord entre expérience et modèle (fig 11b). Par ailleurs, les respirations maximales ajustées pour chaque taille sont faiblement mais significativement différentes $\left(5,9410^{-6} \mathrm{~mol} \mathrm{~m}^{-3} \mathrm{~s}^{-1}\right.$ pour les agrégats de $20-25 \mathrm{~mm} ; 6,410^{-6} \mathrm{~mol} \mathrm{~m}^{-3} \mathrm{~s}^{-1}$ pour les agrégats de $10-12 \mathrm{~mm}$; et $7,1710^{-6}$ $\mathrm{mol} \mathrm{m}-3 \mathrm{~s}^{-1}$ pour les agrégats de $6-8 \mathrm{~mm}$ ).

\section{Distribution des concentrations en $\mathrm{O}_{2}$ au sein des agrégats}

Quelques profils d' $\mathrm{O}_{2}$ mesurés dans des agrégats naturels et dans des agrégats de sol remanié de différentes tailles sont présentés dans la figure 13. La diminution de la concentration d' $\mathrm{O}_{2}$ en fonction de la profondeur est plus régulière 


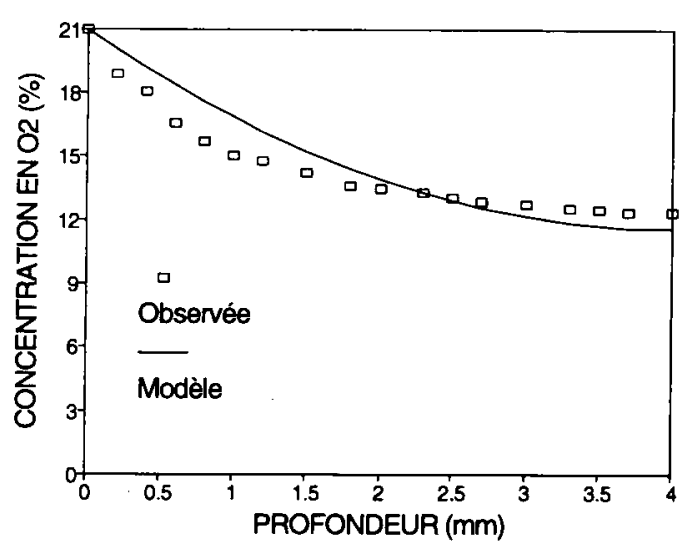

a/

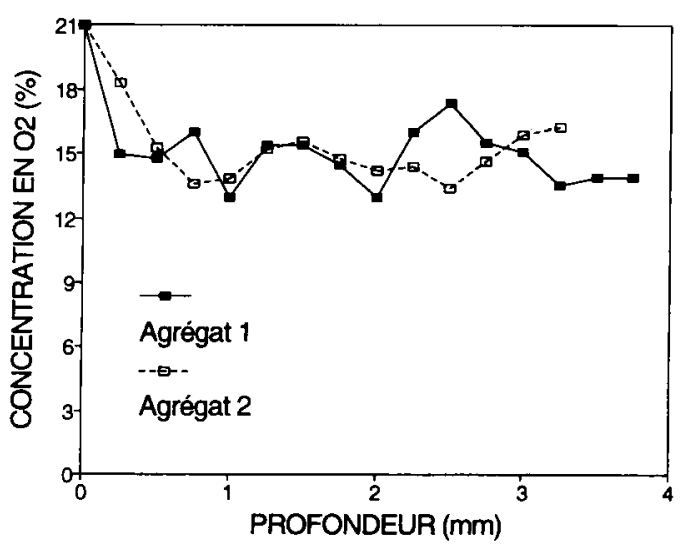

cl

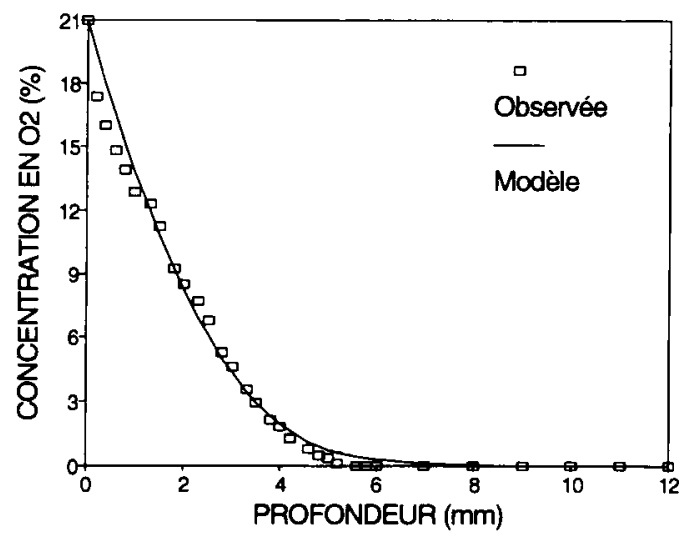

b/

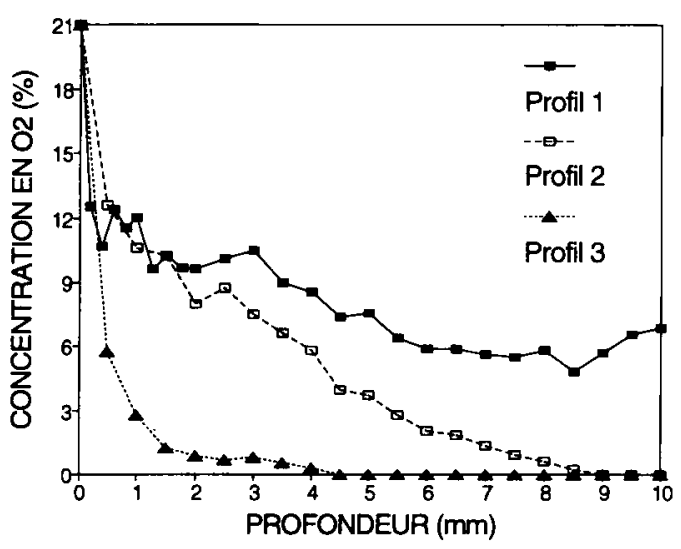

d/

Fig 13. Profils d' $\mathrm{O}_{2}$ observés pour différents agrégats. $\mathbf{a}$ et $\mathbf{b}$ : agrégats de sol remanié (le modèle est confronté aux données expérimentales); $\mathbf{c}$ et $\mathbf{d}$ : agrégats naturels.

dans les agrégats de sol remaniés que dans les agrégats naturels. Pour ces derniers, les irrégularités pourraient être liées aux hétérogénéités précédement évoquées (diffusion, matières organiques).

On constate aussi que la diminution de la concentration d' $\mathrm{O}_{2}$ à la périphérie des agrégats est plus marquée dans les agrégats naturels que dans les agrégats de sol remanié.

La comparaison des profils observés et des profils simulés, avec prise en compte des effets du $\mathrm{CO}_{2}$, montre une surestimation systématique de la concentration en $\mathrm{O}_{2}$ à la périphérie des agrégats et une sous-estimation à leur centre. Cela pourrait être lié à l'existence de corrélations entre l'importance des populations microbiennes et les concentrations en $\mathrm{O}_{2}$ et en $\mathrm{CO}_{2}$.

\section{CONCLUSION}

Dans ce travail, nous avons montré que, par une modélisation simplifiée, nous arrivions à retrouver les grandes tendances des relations proposées entre dénitrification et teneur en eau (Rolston et al, 1984 ; Grundmann et Rolston, 1987 ; Sexstone et al, 1988). Cette similitude montre que l'eau pourrait agir sur la dénitrification essentiellement par l'intermédiaire du volume anoxique qu'elle contrôle comme cela avait déjà été mis en évidence expérimentalement (Sexstone et al, 1988). Cet accord suppose que l'on tienne compte de la structure du sol (densité, granulométrie de mottes et d'agrégats, contacts entre mottes) et du positionnement de l'eau dans l'espace poral interagrégats. En particulier, l'influence des surfaces bloquées à la périphérie 
des agrégats, conséquence des contacts entre agrégats et de leur recouvrement partiel par de l'eau, doit être prise en compte au contraire de la plupart des études précédentes. Leffelaar (1979) n'ignorait pas ces contacts, mais il supposait qu'un blocage donné était équivalent à une décroissance proportionnelle du coefficient de diffusion intra-agrégat. II est possible de montrer que les effets sur l'anoxie (et donc, probablement, sur la dénitrification) de la teneur en eau, de la température et de la structure du sol sont des effets de type interactifs. L'accroissement de la dénitrification avec la température dépend de l'accroissement de l'activité des microorganismes dénitrifiants avec cette température mais aussi de l'accroissement du volume anoxique où l'activité de ces microorganismes va pouvoir s'exprimer. L'accroissement du volume anoxique avec la température dépend de la teneur en eau du sol. Enfin, il existe un effet structure du sol qui n'est jamais pris en compte dans les études relatives à la dénitrification.

L'expérience montre que les agrégats naturels se caractérisent par des hétérogénéités intraagrégats (diffusion de l' $\mathrm{O}_{2}$ et distribution des matières organiques) et interagrégats (respiration microbienne). Quel que soit le type d'agrégats, la respiration microbienne dépend simultanément de $\mathrm{I}^{\prime} \mathrm{O}_{2}$ et du $\mathrm{CO}_{2}$.

Lorsque l'on travaille sur des agrégats de sol remanié (afin d'éliminer les hétérogénéités d'espace poral et de distribution des matières organiques non solubles au sein des agrégats), le modèle au niveau de l'agrégat reflète bien l'expérience si l'on tient compte de la dépendance de la respiration à la concentration en $\mathrm{O}_{2}$ et si l'on y inclut le transfert et les effets liés au $\mathrm{CO}_{2}$. L'analyse des profils de concentration en $\mathrm{O}_{2}$ au sein des agrégats de sol remanié montre qu'il existe néanmoins encore un écart entre le modèle et l'expérience (surestimation de la concentration en $\mathrm{O}_{2}$ à la périphérie de l'agrégat, sous-estimation au centre de l'agrégat). Cet écart pourrait être dû à notre mauvaise connaissance des processus associés au $\mathrm{CO}_{2}$ (coefficients de diffusion de $\mathrm{CO}_{2}$ et de $\mathrm{HCO}_{3}{ }^{-}$, production de $\mathrm{CO}_{2}$ en conditions d'anoxie) et à des dépendances éventuelles de la croissance microbienne à la concentration en $\mathrm{O}_{2}$ et, éventuellement, à la concentration en $\mathrm{CO}_{2}$. II nous reste à tester ces hypothèses.

Notre travail se poursuivra dans 2 voies:

- un retour à l'étude de l'anoxie dans les agrégats naturels avec prise en compte des hétérogénéités de structure et de distribution des matières organiques;
- un travail sur la dénitrification proprement dite avec description de l'activité microbienne au sein des microsites anoxiques et des transferts liés à cette activité microbienne (transferts de nitrate, d'oxyde nitreux, etc).

Le modèle proposé actuellement sera modifié pour tenir compte, aux différentes échelles abordées, des transferts de $\mathrm{CO}_{2}$. II est probable que l'ajout de cette fonction aura des conséquences qui dépendront de la structure du sol (les écarts au modèle actuel étant maximaux pour des granulométries de mottes grossières).

\section{REMERCIEMENTS}

Ce travail a été financé par le programme français Agrotech (AGI.3 physique et microbiologie) de l'INRA et par l'AIP (Travail du sol) du même Institut. Nous remercions Niels Peter Revsbech et les personnes de son équipe (université d'Aarhus, Danemark) qui ont bien voulu accueillir l'un d'entre nous pour le former à la fabrication des microélectrodes à $\mathrm{O}_{2}$.

\section{RÉFÉRENCES}

Arah JRM, Smith KA (1989) Steady-state denitrification in aggregated soils: a mathematical model. $J$ Soil Sci 40, 139-149

Aulakh MS, Rennie DA, Paul EA (1982) Gaseous nitrogen losses from cropped and summer fallowed soils. Can J Soil Sci 62, 187-196

Bridge BJ, Rixon AJ (1976) Oxygen uptake and respiratory quotient of field soil cores in relation to their air-filled pore space. J Soil Sci 27, 279-286

Clay DE, Molina JAE, Clapp CE, Linden DR (1985) Nitrogen-tillage residue management. II. Calibration of potential rate of nitrification by model simulation. Soil Sci Soc Am J 49, 322-325

Craswell ET (1978) Some factors influencing denitrification and nitrogen immobilization in a clay soil. Soil Biol Biochem 10, 241-245

Currie JA (1961) Gaseous diffusion in the aeration of aggregated soils. Soil Sci 92, 40-45

Fazzolari E, Mariotti A, Germon JC (1990a) Nitrate reduction in ammonia: a dissimilatory process in Enterobacter amnigenus. Can J Microbiol 36, 779785

Fazzolari E, Mariotti A, Germon JC (1990b) Dissimilatory ammonia production vs denitrification in vitro and in inoculated soil samples. Can $J$ Microbiol 36, 786-793

Fies JC, Stengel P (1981) Densité texturale de sols naturels. I. Méthode de mesure. agronomie 1, 651658 
Firestone MK (1982) Biological denitrification. In : Nitrogen in agricultural soils (FJ Stevenson, ed). Agronomy 22. American Society of Agronomy, Madison, WI, États-Unis

Grant RF (1991) A technique for estimating denitrification rates at different soil temperatures, water contents, and nitrate concentrations. Soil Sci 152 , 41-52

Greenwood DJ, Berry G (1962) Aerobic respiration in soil crumbs. Nature 195, 161-163

Groot JJR, De Willigen P (1991) Simulation of the nitrogen balance in the soil and in a winter wheat crop. Fert Res 27, 261-272

Grundmann GL, Rolston DE (1987) A water function approximation to degree of anaerobiosis associated with denitrification. Soil Sci 144, 437-441

Hansen S, Jensen HE, Nielsen NE, Svendsen $H$ (1991) Simulation of nitrogen dynamics and biomass production in winter wheat using the Danish simulation model DAISY. Fert Res 27, 245-249

Hénault C (1993) Quantification de la dénitrification dans les sols à l'échelle de la parcelle cultivée, à l'aide d'un modèle prévisionnel. Thèse ENSA Montpellier, France

Johnsson H, Berström L, Jansson P, Paustian K (1987) Simulated nitrogen dynamics and losses in a layered agricultural soil. Agric Ecosyst Environ 18, 333-356

Knowles R (1982) Denitrification. Microbiol Rev 46(1), 43-70

Lafolie F (1991) Modelling water flow, nitrogen transport and root uptake including physical non-equilibrium and optimization of the root water potential. Fert Res 27, 215-131

Leffelaar PA (1977) A theoretical approach to calculate the anaerobic volume fraction in aerated soil in view of denitrification. A computer simulation study. Report no 9, Dept of theoretical Production Ecology, Agricultural University, Wageningen, The Netherlands

Leffelaar PA (1979) Simulation of partial anaerobiosis in a model soil in respect to denitrification. Soil Sci $128,110-120$

Linn DM, Doran JW (1984) Effect of water-filled pore space in carbon dioxide and nitrous oxide production in tilled and nontilled soils. Soil Sci Soc Am J 48, 1267-1272

McConnaughey PK, Bouldin DR (1985) Transient microsite models of denitrification. I. Model development. Soil Sci Soc Am J 49, 886-891
Refsgaard JC, Christensen TH, Ammentorp HC (1991) A model for oxygen transport and consumption in the unsaturated zone. J Hydrol 129, 349-369

Renault P, Stengel P (1994) Modeling oxygen diffusion in aggregated soils. 1. Anaerobiosis inside the aggregates. Soil Sci Soc Am J 58 (4) (in press)

Renault P, Sierra J (1994) Modeling oxygen diffusion in aggregated soils. 2. Anaerobiosis in topsoil layers. Soil Sci Soc Am J 58 (4) (in press)

Revsbech NP, Ward DM (1983) Oxygen microelectrode that is insensitive to medium chemical composition: use in an acid microbial mat dominated by Cyanidium caldanium. Appl Environ Microbiol 45, 755-759

Revsbech NP, Jorgensen BB (1986) Microelectrodes: their use in microbial ecology. In : Advances in microbial ecology (KC Marshall, ed), Plenum Press, New York, 293-352

Revsbech NP (1989) An oxygen microelectrode with a guard cathode. Limnol Oceanogr 34, 474-478

Rijtema PE, Kroes JG (1991) Some results of nitrogen simulations with the model ANIMO. Fert Res 27, 189-198

Rolston DE, Rao PSC, Davidson JM, Jessup RE (1984) Simulation of denitrification losses of nitrate fertilizer applied to uncropped, cropped and manureamended field plots. Soil Sci 137 (4), 270-279

Sexstone AJ, Parkin TB, Tiedje JM (1988) Denitrification response to soil wetting in aggregated and unaggregated soil. Soil Biol Biochem 20(5), 767-769

Sexstone AJ, Revsbech NP, Parkin TB, Tiedje JM (1985) Direct measurement of oxygen profiles and denitrification rates in soil aggregates. Soil Sci Soc Am J 49, 645-651

Sierra J (1994) Étude de l'anoxie dans les sols à structure agrégée en relation avec la respiration microbienne. Thèse UCB Lyon I, France

Skopp J (1985) Oxygen uptake and transport in soils: analysis of the air-water interfacial area. Soil Sci Soc Am J 49, 1327-1331

Skopp J, Jawson MD, Doran JW (1990) Steady-state aerobic microbial activity as a function of soil water content. Soil Sci Soc Am J 49, 1327-1331

Smith KA (1980) A model of the extent of anaerobic zones in aggregated soils, and its potential application to estimates of denitrification. J Soil Sci 31, 263-277

Vereecken H, Vanclooster M, Swerts M, Diels J (1991) Simulating water and nitrogen behavior in soils cropped with winter wheat. Fert Res 27, 233-243 